\title{
Mixed convection in non-Newtonian fluids along a vertical plate with variable surface heat flux in a porous medium
}

\author{
Rama Subba Reddy Gorla, M. Kumari
}

\begin{abstract}
A nonsimilar boundary layer analysis is presented for the problem of mixed convection in power-law type non-Newtonian fluids along a vertical plate with power-law surface heat flux distribution. The mixed convection regime is divided into two regions, namely, the forced convection dominated regime and the free convection dominated regime. The two solutions are matched. Numerical results are presented for the details of the velocity and temperature fields. A discussion is provided for the effect of viscosity index on the surface heat transfer rate.

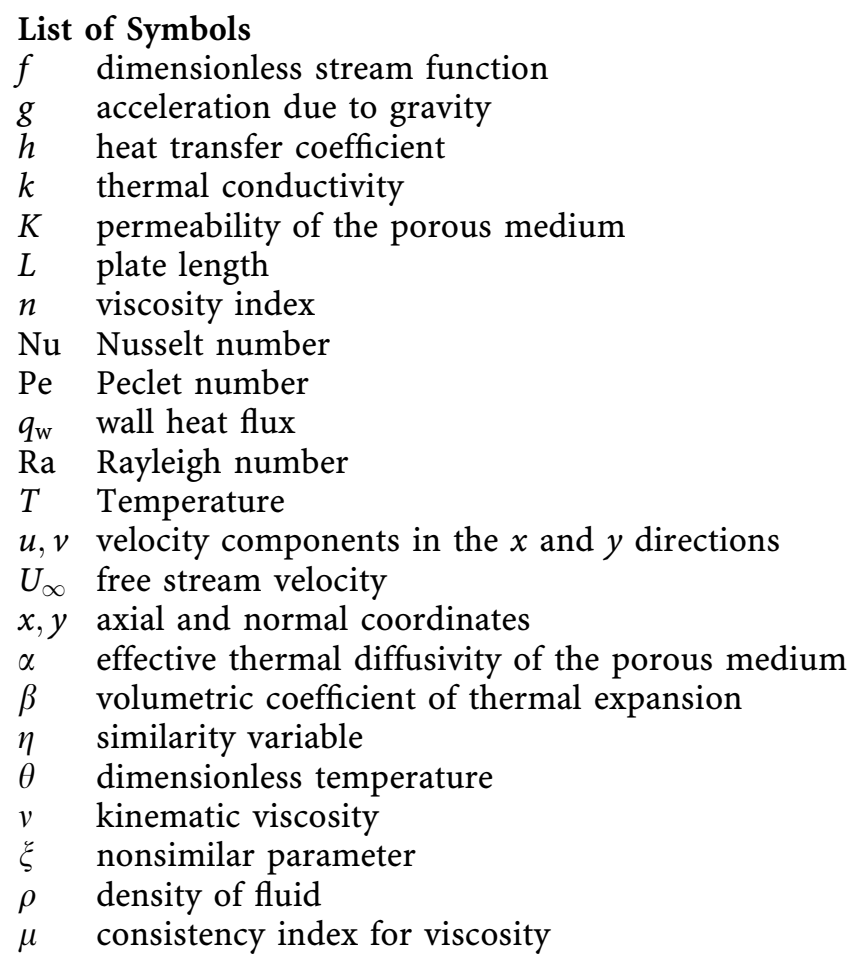

R.S.R. Gorla

Department of Mechanical Engineering

Cleveland State University Cleveland, Ohio 44115, USA

M. Kumari

Department of Mathematics

Indian Institute of Science,

Bangalore 560 012, India $\tau_{\mathrm{w}} \quad$ wall shear stress

$\psi \quad$ stream function

\author{
Subscripts \\ w wall conditions \\ $\infty$ free stream conditions
}

\section{Introduction}

The research outlined in this paper was motivated because of the numerous thermal engineering applications such as geothermal systems, crude oil extraction, thermal insulation and ground water pollution. The similarity solutions for mixed convection from horizontal plates and cylinders in a fluid-saturated porous medium were presented by Cheng (1977). Nakayama and Koyama (1987) considered similarity solutions for two-dimensional and axisymmetric bodies in a porous medium. Cheng and Minkowycz (1977) presented similarity solutions for free convective heat transfer from a vertical plate in a fluid-saturated porous medium. Gorla and Tornabene (1988) solved the nonsimilar problem of free convective heat transfer from a vertical plate embedded in a saturated porous medium with an arbitrarily varying surface heat flux. Hsieh et al. (1993) presented solutions for mixed convection from vertical plates in porous media. All these studies were concerned with Newtonian fluid flows. A number of industrially important fluids including fossil fuels which may saturate underground beds display non-Newtonian behavior. Non-Newtonian fluids exhibit a nonlinear relationship between shear stress and shear rate.

Chen and Chen (1988) presented similarity solutions for free convection of non-Newtonian fluids over vertical surfaces in porous media. Nakayama and Koyama (1991) studied the natural convection over a non-isothermal body of arbitrary shape embedded in a porous medium. The mixed convection from horizontal surfaces in porous media was studied by Kumari et al. (1997). Gorla et al. (1997) investigated the effect of surface mass transfer on mixed convection in non-Newtonian fluids in porous media.

The present work has been undertaken in order to analyze the mixed convection from a vertical plate in nonNewtonian fluid saturated porous media. The power law model of Ostwald-de-Waele, which is adequate for many non-Newtonian fluids, is considered here. The governing equations are first transformed into a dimensionless form and the resulting nonsimilar set of equations is solved by a finite difference method. The boundary condition of variable surface heat flux is treated in this paper. 
Numerical results for the velocity and temperature fields are presented.

\section{Analysis}

Let us consider the mixed convention in a porous medium from an impermeable vertical plate, which is heated and has a variable wall heat flux. The properties of the fluid and the porous medium are assumed to be constant and isotropic. The Darcy model is considered which is valid under conditions of small permeability of porous medium and flow velocity. Also, the slip velocity at the wall is imposed, which has a smaller effect on the heat transfer results as the distance from the leading edge increases. The axial and normal coordinates are $x$ and $y$, and the corresponding flow velocities are $u$ and $v$ respectively. Figure 1 shows the coordinate system and model of the flow. The gravitational acceleration $g$ is acting downwards opposite to the normal coordinate $y$. The governing equations under the Boussinesq and boundary layer approximations are given by,

$\frac{\partial u}{\partial x}+\frac{\partial v}{\partial y}=0$

$u^{n}=U_{\infty}^{n}+K \rho g \beta\left(T-T_{\infty}\right)$

$u \frac{\partial T}{\partial x}+v \frac{\partial T}{\partial y}=\alpha \frac{\partial^{2} T}{\partial y^{2}}$

In the above equations, $T$ is the temperature of the wall; $n$ is the viscosity index; $\rho$ is the density; $K$ is the permeability of the porous medium; $\beta$ is the volumetric coefficient of thermal expansion; $\mu$ is the viscosity; $\alpha$ is the equivalent thermal diffusivity of the porous medium. With power law variation in wall temperature, the boundary conditions can be written as

$y=0 ; v=0, q_{\mathrm{w}}=-k \frac{\partial T}{\partial y}=A x^{\lambda}$

$y=\infty ; u=U_{\infty}, T=T_{\infty}$

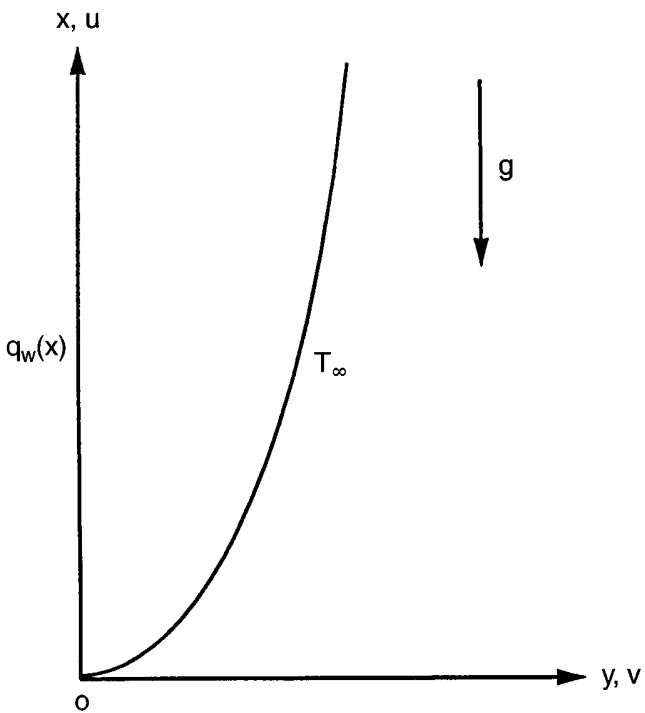

Fig. 1. Flow model and coordinate system where $A$ and $\lambda$ are prescribed constants. Note that $\lambda=0$ corresponds to the case of uniform wall heat flux.

\section{A. Forced convection dominated regime}

The continuity equation is automatically satisfied by defining a stream function $\psi(x, y)$ such that

$u=\frac{\partial \psi}{\partial y}$ and $v=-\frac{\partial \psi}{\partial x}$

Proceeding with the analysis, we define the following transformations:

$\eta=\frac{y}{x} \mathrm{Pe}_{x}^{1 / 2}$

$\psi=\alpha \operatorname{Pe}_{x}^{1 / 2} f\left(\xi_{f}, \eta\right)$

$\xi_{f}=\mathrm{Ra}_{x}^{* n} / \mathrm{Pe}_{x}^{(2 n+1) / 2}$

$\theta=\frac{\left(T-T_{\infty}\right) \mathrm{Pe}_{x}^{\frac{1}{2}}}{\left(\frac{q_{\mathrm{w}} x}{k}\right)}$

$\mathrm{Pe}_{x}=\frac{U_{\infty} x}{\alpha}$

$\mathrm{Ra}_{x}^{*}=\frac{x}{\alpha}\left(\frac{\rho K g \beta q_{\mathrm{w}} x}{\mu k}\right)^{1 / n}$

The governing equations and boundary conditions, Eqs. (1)-(4), can then be transformed into

$\left(f^{\prime}\right)^{n}=1+\xi \theta$

$\theta^{\prime \prime}-\left(\frac{2 \lambda+1}{2}\right) f^{\prime} \theta+\frac{1}{2} f \theta^{\prime}$

$=\left(\frac{1+2 \lambda}{2}\right) \xi_{f}\left[f^{\prime} \frac{\partial \theta}{\partial \xi_{f}}-\theta^{\prime} \frac{\partial f}{\partial \xi_{f}}\right]$

$\frac{1}{2} f\left(\xi_{f}, 0\right)+\left(\frac{1+2 \lambda}{2}\right) \xi_{f} \frac{\partial f}{\partial \xi_{f}}\left(\xi_{f}, 0\right)=0$ or

$$
\begin{array}{lr}
f\left(\xi_{f}, 0\right)=0, & \theta^{\prime}\left(\xi_{f}, 0\right)=-1, \\
f^{\prime}\left(\xi_{f}, \infty\right)=1, & \theta\left(\xi_{f}, \infty\right)=0
\end{array}
$$

The primes in the above equations denote partial differentiations with respect to $\eta$.

In the above system of equations, the dimensionless parameter $\xi_{f}$ is a measure of the buoyancy effect on forced convection. The case of $\xi_{f}=0$ corresponds to pure forced convection. The limiting case of $\xi_{f}=\infty$ corresponds to pure free convection regime. The Eqs. (6)-(8) cannot be solved for the entire regime of mixed convection because of the singularity at $\xi_{f}=\infty$. The above system of equations is used to solve the region covered by $\xi_{f}=0-1$ to provide the first half of the total solution of the mixed convection regime.

Some of the physical quantities of interest include the velocity components $u$ and $v$ in the $x$ and $y$ directions, the local friction factor $C_{f x}$ (defined as $\tau_{\mathrm{w}} /\left[\left(\rho U_{\infty}^{2}\right) / 2\right]$ where $\left.\tau_{\mathrm{w}}=\mu(\partial u / \partial y)_{y=0 .}^{n}\right)$ and the local Nusselt number $\mathrm{Nu}_{x}=$ $h x / k$, where $h=q_{\mathrm{w}} /\left[T_{\mathrm{w}}(x)-T_{\infty}\right]$. They are given by

$u=U_{\infty} f^{\prime}\left(\xi_{f}, \eta\right)$ 


$$
\begin{aligned}
\begin{array}{r}
v=-\alpha\left(\frac{U_{\infty}}{x^{m} \alpha}\right)^{\frac{1}{2}} x^{\frac{m-1}{2}}\left(\frac{1}{2} f\left(\xi_{f}, \eta\right)-\frac{1}{2} \eta f^{\prime}\left(\xi_{f}, \eta\right)\right. \\
\left.+\left(\frac{1+2 \lambda}{2}\right) \xi_{f} \frac{\partial f}{\partial \xi_{f}}\right)
\end{array} \\
C_{f x}=\frac{2 \mathrm{Pe}_{x}^{n / 2}}{\operatorname{Re}_{x}}\left[f^{\prime \prime}\left(\xi_{f}, 0\right)\right]^{n}
\end{aligned}
$$

where

$\operatorname{Re}_{x}=\left(\frac{U_{\infty}^{2-n} x^{n}}{v}\right)$

$\mathrm{Nu}_{x}=\frac{\mathrm{Pe}_{x}^{\frac{1}{2}}}{\theta\left(\xi_{f}, 0\right)}$

\section{B. Free convection dominated regime}

For buoyancy dominated regime the following dimensionless variables are introduced in the transformation

$\eta=\frac{y}{x}\left(\mathrm{Ra}_{x}^{*}\right)^{n /(2 n+1)}$

$\xi_{n}=\frac{\mathrm{Pe}_{x}}{\mathrm{Ra}_{x}^{* 2 n /(2 n+1)}}$

$\psi=\alpha\left(\mathrm{Ra}_{x}^{*}\right)^{n /(2 n+1)} f\left(\xi_{n}, \eta\right)$

$\theta\left(\xi_{n}, \eta\right)=\frac{\left(T-T_{\infty}\right) \mathrm{Ra}_{x}^{* n /(2 n+1)}}{\left(\frac{q_{w} x}{k}\right)}$

Substituting Eqs. (14) and (15) into the governing Eqs. (1)-(4) leads to

$$
\begin{aligned}
& \left(f^{\prime}\right)^{n}=\xi^{n}+\theta \\
& \theta^{\prime \prime}+\frac{\lambda+n+1}{(2 n+1)} f \theta^{\prime}-\frac{n(2 \lambda+1)}{(2 n+1)} f^{\prime} \theta \\
& =-\left(\frac{2 \lambda+1}{2 n+1}\right) \xi_{n}\left[f^{\prime} \frac{\partial \theta}{\partial \xi_{n}}-\theta^{\prime} \frac{\partial f}{\partial \xi_{n}}\right] \\
& \left(\frac{\lambda+n+1}{2 n+1}\right) f\left(\xi_{n}, 0\right)-\left(\frac{2 \lambda+1}{(2 n+1)}\right) \xi_{n} \frac{\partial f}{\partial \xi_{n}}\left(\xi_{n}, 0\right)=0 \\
& \quad \text { or } \quad f\left(\xi_{n}, 0\right)=0, \\
& \quad \theta^{\prime}\left(\xi_{n}, 0\right)=-1, \quad f^{\prime}\left(\xi_{n}, \infty\right)=\xi_{n}, \quad \theta\left(\xi_{n}, \infty\right)=0
\end{aligned}
$$

and the primes in Eqs. (16)-(18) denote partial differentiations with respect to $\eta$.

Note that the $\xi_{n}$ parameter here represents the forced flow effect on free convection. The case of $\xi_{n}=0$ corresponds to pure convection and the limiting case of $\xi_{n}=\infty$ corresponds to pure forced convection. The above systems of Eqs. (16)-(18) is solved over the region covered by $\xi_{n}=0-1$ to provide the other half of the solution for the entire mixed convection regime.

The velocity components $u$ and $v$, the local friction factor and the local Nusselt number for this case have the following expressions

$u=\alpha\left(C_{1}\right)^{2 n /(2 n+1)} \cdot x^{(2 \lambda+1) /(2 n+1)} \cdot f^{\prime}\left(\xi_{n}, \eta\right)$

$$
\begin{aligned}
& v=-\alpha\left(C_{1}\right)^{n /(2 n+1)} \\
& \left(\frac{\lambda+n+1}{(2 n+1)} f\left(\xi_{n}, \eta\right)+\frac{\lambda-n}{(2 n+1)} \eta f^{\prime}\left(\xi_{n}, \eta\right)\right. \\
& \left.-\frac{2 \lambda+1}{(2 n+1)} \xi_{n} \frac{\partial f}{\partial \xi_{n}}\right) \\
& C_{f x}=\frac{2 \mu}{\rho U_{\infty}^{2}} \alpha^{n} C_{1}^{3 n^{2} /(2 n+1)} \cdot x^{(3 \lambda-n+1) n /(2 n+1)}\left[f^{\prime \prime}\left(\xi_{n}, 0\right)\right]^{n}
\end{aligned}
$$

$\mathrm{Nu}_{x}=\frac{\mathrm{Ra}_{x}^{* n /(2 n+1)}}{\theta\left(\xi_{n}, 0\right)}$

where

$C_{1}=\mathrm{Ra}_{x}^{*} / x^{(n+\lambda+1) / n}$

\section{Numerical scheme}

The numerical scheme to solve Eqs. (6) and (7) adopted here is based on a combination of the following concepts:

(a) The boundary conditions for $\eta=\infty$ are replaced by

$f^{\prime}\left(\xi, \eta_{\max }\right)=1, \quad \theta\left(\xi, \eta_{\max }\right)=0$

where $\eta_{\max }$ is a sufficiently large of $\eta$ at which the boundary conditions (8) are satisfied. $\eta_{\max }$ varies with the value of $n$. In the present work, a value of $\eta_{\max }=25$ was checked to be sufficient for free stream behavior.

(b) The two-dimensional domain of interest $(\xi, \eta)$ is discretized with an equispaced mesh in the $\xi$-direction and another equispaced mesh in the $\eta$-direction.

(c) The partial derivatives with respect to $\eta$ are evaluated by the second order difference approximation.

(d) Two iteration loops based on the successive substitution are used because of the nonlinearity of the equations.

(e) In each inner iteration loop, the value of $\xi$ is fixed while each of the Eqs. (6) and (7) is solved as a linear second order boundary value problem of ODE on the $\eta$-domain. The inner iteration is continued until the nonlinear solution converges with a convergence criterion of $10^{-6}$ in all cases for the fixed value of $\xi$.

(f) In the outer iteration loop, the value of $\xi$ is advanced. The derivatives with respect to $\xi$ are updated after every outer iteration step.

Table $1 \mathbf{a}, \mathbf{b}$; Comparison of values of $1 / \theta\left(\xi_{F}, 0\right)$ for $n=1$, and $\xi_{F}=0$. Comparison of values of $1 / \theta\left(\xi_{N}, 0\right)$ for $n=1$, and $\xi_{N}=0$

\begin{tabular}{ccc}
\hline$\lambda$ & Present results & Hsieh et al. \\
\hline $1 / \theta\left(\xi_{F}, 0\right)$ & & \\
-0.5 & 0.56414 & 0.5642 \\
0.0 & 0.88602 & 0.8863 \\
0.5 & 1.12812 & 1.1284 \\
1.0 & 1.32901 & 1.3294 \\
$1 / \theta\left(\xi_{N}, 0\right)$ & & \\
-0.5 & 0.58106 & 0.5818 \\
0.0 & 0.77111 & 0.7715 \\
0.5 & 0.89962 & 0.8998 \\
1.0 & 0.99999 & 1.0000 \\
\hline
\end{tabular}


In the inner iteration step, the finite difference approximation for Eqs. (6) and (7) is solved as a boundary value problem. We consider Eq. (6) first. By defining $f=\phi$, Eq. (6) may be written in the form

Table 2. Values of $1 / \theta\left(\xi_{F}, 0\right)$ and $1 / \theta\left(\xi_{N}, 0\right)$ for $\lambda=0.5$

\begin{tabular}{llll}
\hline$\xi_{F}$ & $n=0.5$ & $n=1.0$ & $n=1.5$ \\
\hline $1 / \theta\left(\xi_{F}, 0\right)$ & & & \\
0.0 & 1.12812 & 1.12812 & 1.12812 \\
0.1 & 1.18530 & 1.15694 & 1.14738 \\
0.2 & 1.23781 & 1.18372 & 1.16536 \\
0.3 & 1.28664 & 1.20880 & 1.18225 \\
0.4 & 1.33247 & 1.23246 & 1.19821 \\
0.5 & 1.37580 & 1.25488 & 1.21334 \\
0.6 & 1.41700 & 1.27622 & 1.22776 \\
0.7 & 1.45635 & 1.29661 & 1.24154 \\
0.8 & 1.49408 & 1.31615 & 1.25475 \\
0.9 & 1.53039 & 1.33494 & 1.26744 \\
1.0 & 1.56542 & 1.35304 & 1.27966 \\
\hline$\xi_{N}$ & & & \\
\hline $1 / \theta\left(\xi_{N}, 0\right)$ & & & \\
1.0 & 1.56540 & 1.35303 & 1.27967 \\
0.9 & 1.48224 & 1.28955 & 1.22543 \\
0.8 & 1.45059 & 1.25790 & 1.19329 \\
0.7 & 1.41101 & 1.22044 & 1.15719 \\
0.6 & 1.36526 & 1.17948 & 1.11978 \\
0.5 & 1.31395 & 1.13621 & 1.08253 \\
0.4 & 1.25693 & 1.09130 & 1.04657 \\
0.3 & 1.19314 & 1.04519 & 1.01295 \\
0.2 & 1.12001 & 0.99822 & 0.98303 \\
0.1 & 1.03007 & 0.95077 & 0.95882 \\
0.0 & 0.85144 & 0.90345 & 0.94534 \\
\hline & & &
\end{tabular}

Table 3. Values of $1 / \theta\left(\xi_{F}, 0\right)$ and $1 / \theta\left(\xi_{N}, 0\right)$ for $n=1.5$ and $\lambda=0.5$

\begin{tabular}{lllllll}
\hline$\xi_{F}$ & $\lambda=-0.5$ & $\lambda=0.0$ & $\lambda=0.5$ & $\lambda=1.0$ & $\lambda=1.5$ & $\lambda=2.0$ \\
\hline $1 / \theta\left(\xi_{F}, 0\right)$ & & & & & \\
0.0 & 0.56414 & 0.88602 & 1.12812 & 1.32901 & 1.50410 & 1.66121 \\
0.1 & 0.58379 & 0.90534 & 1.14738 & 1.34827 & 1.52337 & 1.68050 \\
0.2 & 0.60106 & 0.92308 & 1.16536 & 1.36642 & 1.54164 & 1.69888 \\
0.3 & 0.61655 & 0.93951 & 1.18225 & 1.38360 & 1.55905 & 1.71645 \\
0.4 & 0.63067 & 0.95486 & 1.19821 & 1.39995 & 1.57567 & 1.73330 \\
0.5 & 0.64368 & 0.96930 & 1.21334 & 1.41554 & 1.59161 & 1.74950 \\
0.6 & 0.65678 & 0.98293 & 1.22776 & 1.43047 & 1.60692 & 1.76510 \\
0.7 & 0.66710 & 0.99588 & 1.24154 & 1.44481 & 1.62166 & 1.78016 \\
0.8 & 0.67776 & 1.00821 & 1.25475 & 1.45860 & 1.63588 & 1.79473 \\
0.9 & 0.68784 & 1.01999 & 1.26744 & 1.47189 & 1.64963 & 1.80883 \\
1.0 & 0.69743 & 1.03129 & 1.27966 & 1.48474 & 1.66294 & 1.82252 \\
\hline$\xi_{N}$ & & & & & & \\
\hline $1 / \theta\left(\xi_{N}, 0\right)$ & & & & & \\
1.0 & 0.69744 & 1.03128 & 1.27967 & 1.48473 & 1.66296 & 1.82250 \\
0.9 & 0.68011 & 0.99471 & 1.22311 & 1.41067 & 1.57322 & 1.71851 \\
0.8 & 0.66451 & 0.96939 & 1.19121 & 1.37285 & 1.53000 & 1.67030 \\
0.7 & 0.64931 & 0.94205 & 1.15539 & 1.32975 & 1.48034 & 1.61462 \\
0.6 & 0.63463 & 0.91455 & 1.11826 & 1.28443 & 1.42769 & 1.55524 \\
0.5 & 0.62062 & 0.88774 & 1.08125 & 1.23870 & 1.37415 & 1.49452 \\
0.4 & 0.60747 & 0.86221 & 1.04543 & 1.19398 & 1.32139 & 1.43436 \\
0.3 & 0.59544 & 0.83853 & 1.01181 & 1.15160 & 1.27107 & 1.37668 \\
0.2 & 0.58490 & 0.81748 & 0.98160 & 1.11320 & 1.22519 & 1.32383 \\
0.1 & 0.57644 & 0.80034 & 0.95673 & 1.08132 & 1.18682 & 1.27939 \\
0.0 & 0.57164 & 0.79047 & 0.94222 & 1.06253 & 1.16404 & 1.25282 \\
\hline
\end{tabular}

$a_{1} \phi^{\prime}+b_{1} \phi=S_{1}$

where

$$
\begin{aligned}
& a_{1}=\left(\phi^{\prime}\right)^{n-1} \\
& b_{1}=0 \\
& S_{1}=1+\xi \theta
\end{aligned}
$$

The coefficients $a_{1}, b_{1}$ and the source term in Eq. (25) in the inner iteration step are evaluated by using the solution from the previous iteration step. Equation (25) is then transformed to a finite difference equation by applying the central difference approximations to the first and second derivatives. The finite difference equations form a tridiagonal system and can be solved by the tridiagonal solution scheme.
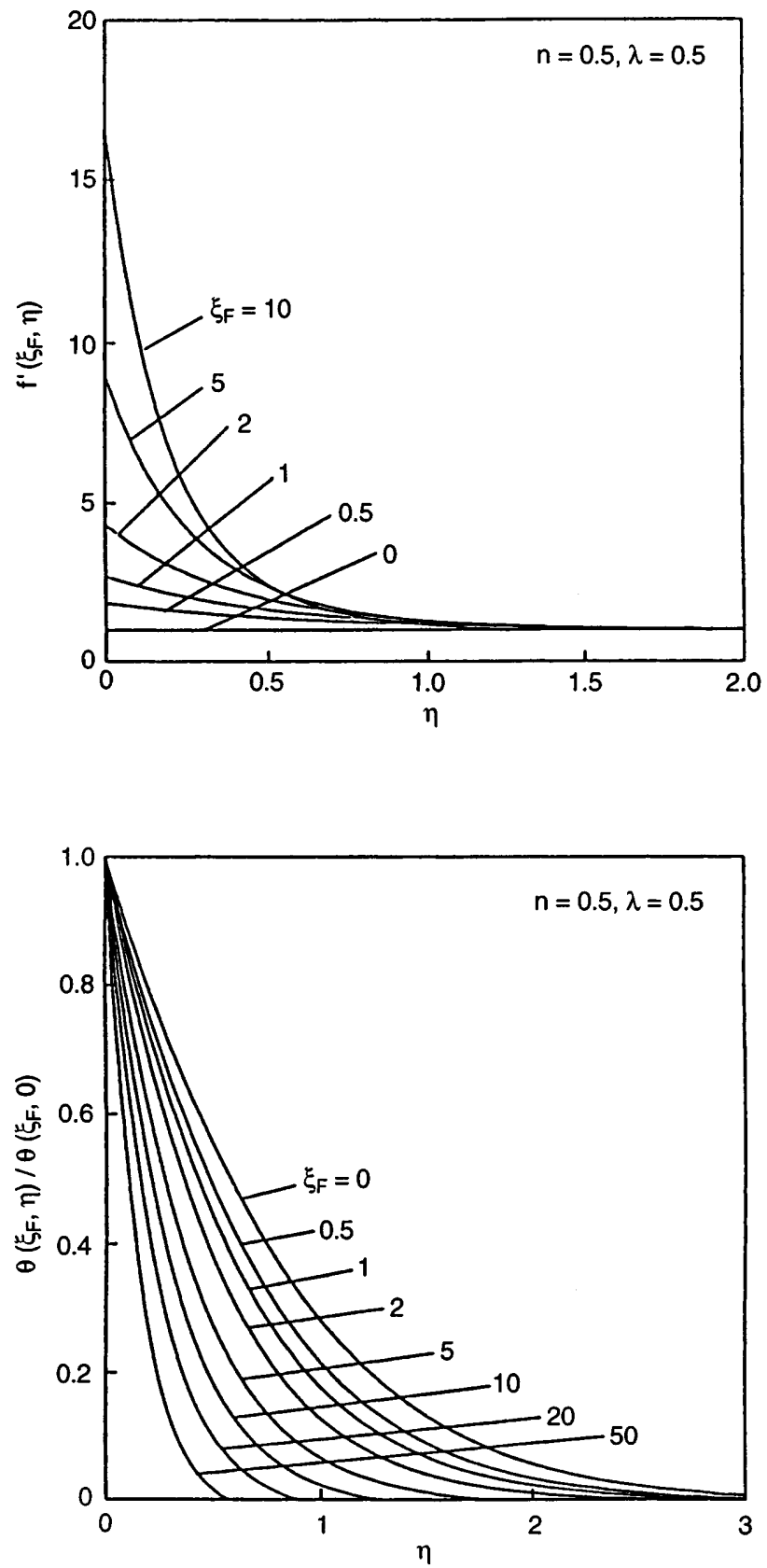

Fig. 2. Velocity and temperature profiles for $n=0.5, \lambda=0.5$ 
Equation (7) is also written as a second-order boundary value problem similar to Eq. (26), namely

$a_{2} \theta^{\prime \prime}+b_{2} \theta^{\prime}+c_{2} \theta=S_{2}$

where

$$
\begin{aligned}
& a_{2}=1 \\
& b_{2}=\frac{1}{2} \phi \\
& c_{2}=-\lambda \phi^{\prime} \\
& S_{2}=\left(\frac{1+2 \lambda}{2}\right) \xi\left[\phi^{\prime} \frac{\partial \theta}{\partial \xi}-\theta^{\prime} \frac{\partial \phi}{\partial \xi}\right]
\end{aligned}
$$

The gradients $\partial \theta / \partial \xi$ and $\partial \phi / \partial \xi$ were evaluated to a firstorder finite difference approximation using the present value of $\xi$ (unknown) and the previous value of $\xi-\Delta \xi$
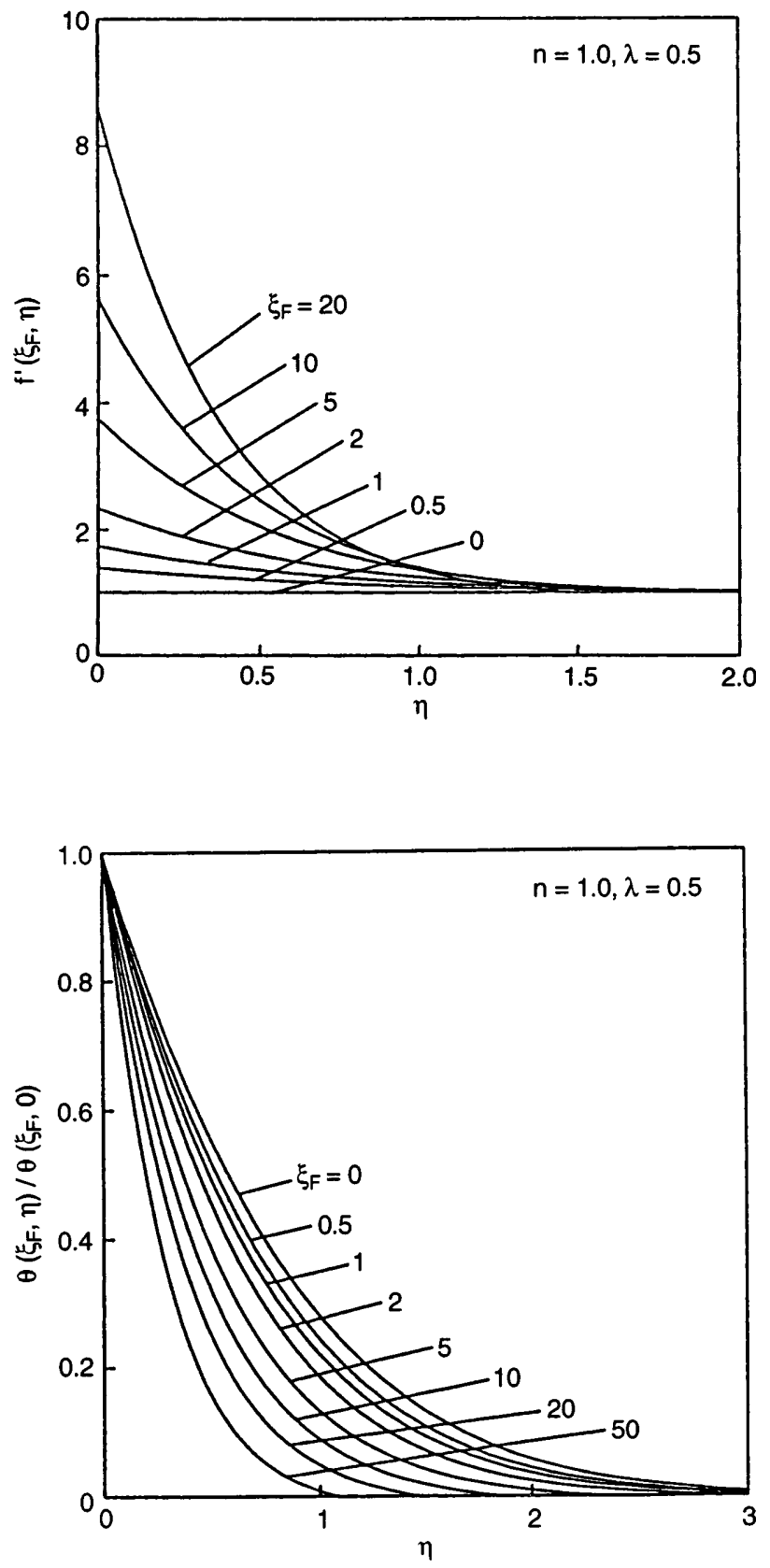

Fig. 3. Velocity and temperature profiles for $n=1.0, \lambda=0.5$ (known), with the unknown present value moved to the left hand side of Eq. (27).

The numerical results are affected by the number of mesh points in both directions. To obtain accurate results, a mesh sensitivity study was performed. After some trials, in the $\eta$-direction 190 mesh points were chosen whereas in the $\xi$-direction, 41 mesh points were used. The tolerance for convergence was $10^{-6}$. Increasing the mesh points to a larger value led to identical results.

The two systems of partial differential Eqs. (6)-(8) and (16)-(18) have similar form. Thus, they were solved using the procedure described above. The complete solution for the entire mixed convection regime was constructed from the two separate solutions of these two set of equations.
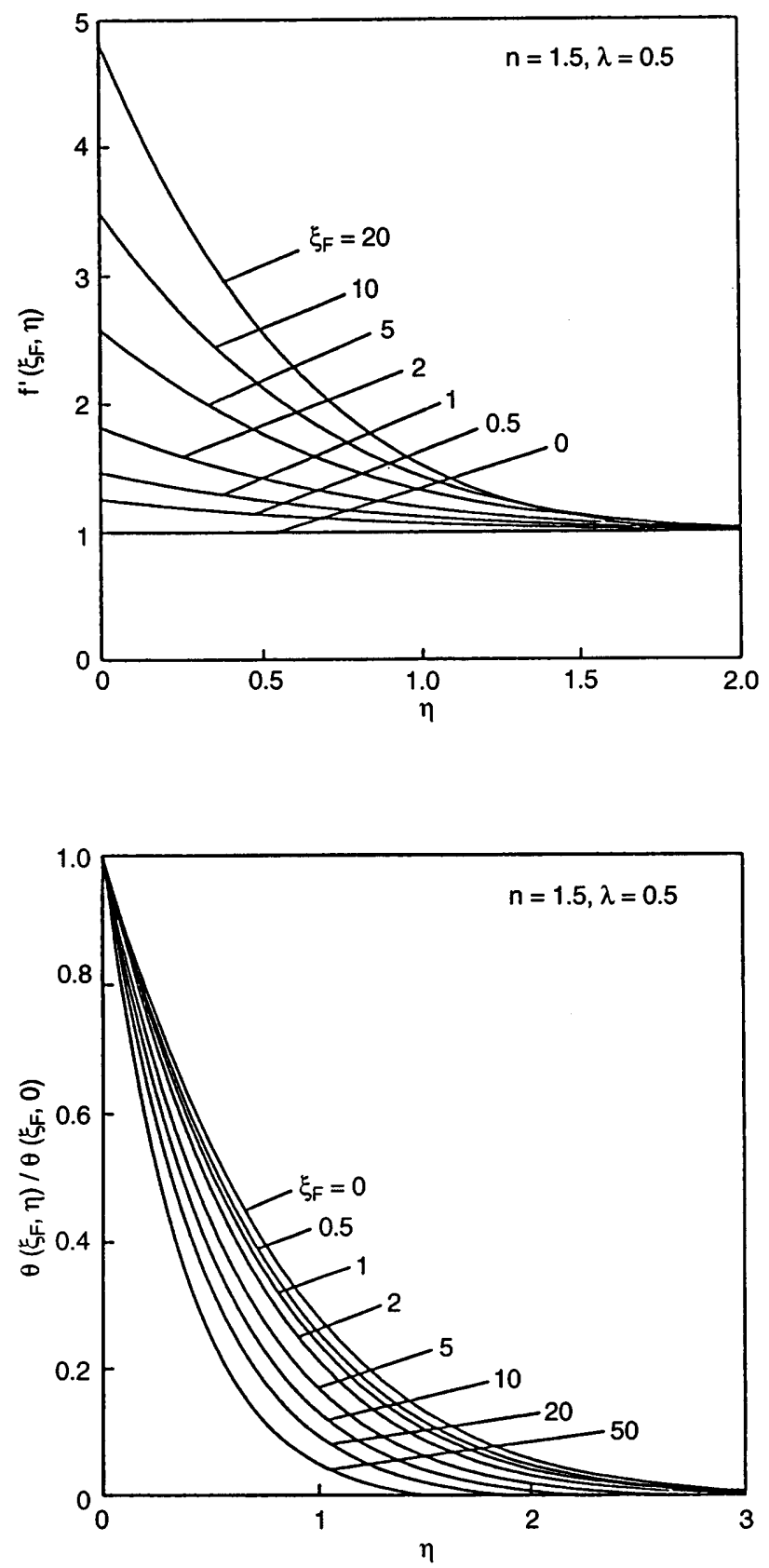

Fig. 4. Velocity and temperature profiles for $n=1.5, \lambda=0.5$ 


\section{Results and discussion}

Numerical results for $1 / \theta(\xi, 0)$ are tabulated in Tables $1-3$. In order to assess the accuracy of the numerical results, we compare our results for Newtonian fluid $(n=1)$ with those of Hsieh et al. (1993). The agreement between the two is within $0.01 \%$ difference. Therefore, the present results are highly accurate. The velocity and temperature profiles are displayed in Figs. 2-5 for a range of values of $n, \lambda$ and $\xi_{f}$. The thermal boundary layer thickness increases as $\xi_{f}$ increases. The slip velocity at the porous surface $f^{\prime}(\xi, 0)$ decreases as the viscosity index $n$ increases. As the temperature exponent $\lambda$ increases, the momentum and thermal boundary layer thicknesses decrease. The inverse of the surface temperature and hence the heat transfer rate increases as $\xi_{f}$ increases. The slip velocity at the wall increases of $\xi_{f}$ or $m$ increases.

Figure 6 displays the variation of Nusselt number with $\xi_{f}$ for $n=0.5$ and 1.5. It is observed that the solutions for the forced convection dominated regime and the free convection dominated regime meet and match over the mixed convection regime. As $\lambda$ and $\xi_{f}$ increase, the Nusselt number increases for a given $n$. As $n$ increases, the Nusselt number decreases. The domain for pure forced convection, mixed convection and pure free convection may be established from the present results based upon 5\% difference in the Nusselt number from pure forced to pure free convection limit.
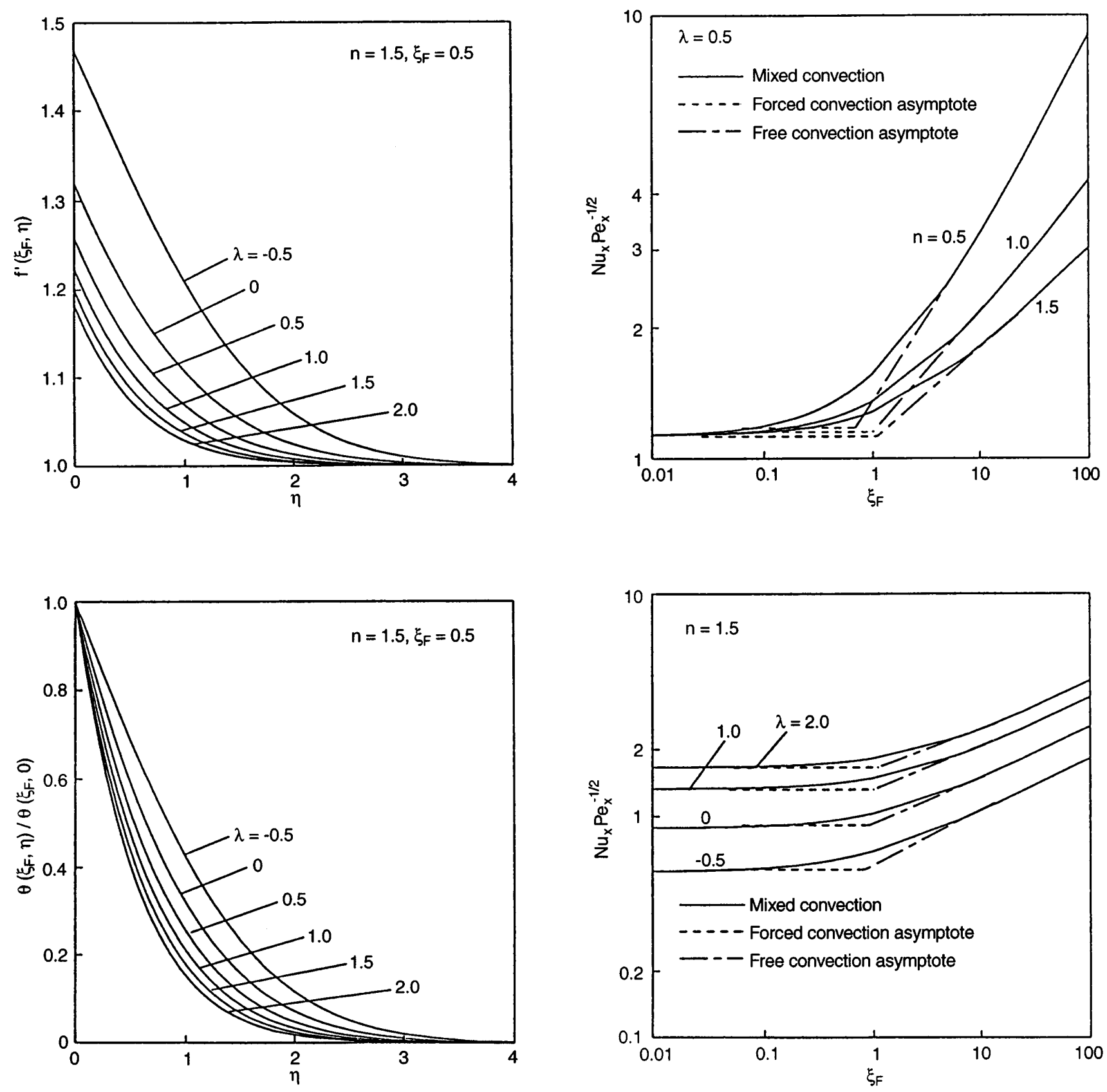

Fig. 5. Velocity and temperature profiles for $n=1.5, \xi_{F}=0.5$

Fig. 6. Local Nusselt number 


\section{Concluding remarks}

In this paper, we have presented a boundary layer analysis for the mixed convection in non-Newtonian fluids along a non-isothermal vertical plate embedded in fluid-saturated porous medium. The flow regime was divided into forced convection dominated and natural convection dominated regions. In the forced convection dominated region, $\xi_{f}=\mathrm{Ra}_{x}^{* n} / \mathrm{Pe}_{x}^{(2 n+1) / 2}$ characterizes the buoyancy effect on forced convection where as $\xi_{n}=\mathrm{Pe}_{x} / \mathrm{Ra}_{x}^{* 2 n /(2 n+1)}$ is a measure of the effect of forced flow on free convection. Numerical solutions using a finite difference scheme were obtained for the flow and temperature fields. The viscosity index, $n$ was varied from $0.5-1.5$.

\section{References}

Chen HT; Chen CK (1988) Free Convection of Non- Newtonian Fluids Along a Vertical Plate Embedded in a Porous Medium. Trans ASME J Heat Trans. 110: 257-260

Cheng P (1977) Similarity Solutions For Mixed Convection From Horizontal Impermeable Surfaces in Saturated Porous Media. Int J Heat and Mass Transfer 20: 893-898

Cheng P; Minkowycz WJ (1977) Free Convection about a Vertical Flat plate Embedded in a Porous Medium With Application to Heat Transfer From a Dike. J Geophys Research 82: 20402049

Gorla RSR; Tornabene R (1988) Free convection from a Vertical Plate With Nonuniform Surface Heat Flux and Embedded in a Porous Medium. Transport in Porous Media J 3: 95-106

Gorla RSR; Takhar HS (1997) Mixed Convection in Non-Newtonian Fluids Along a Vertical Plate in Porous Media with Surface Mass Transfer. Int J Num Meth Heat and Fluid Flow 7: 596-608

Hsieh JC; Chen TS; Armaly BF (1993) Nonsimilarity Solutions For Mixed Convection From Vertical Surfaces in Porous Media. Int Heat and Mass Trans J 36: 1485-1493

Kumari M; Gorla RSR; Byrd L (1997) Mixed Convection in NonNewtonian Fluids Along a Horizontal Plate in a Porous Medium. Trans ASME J Energy Resources Technol 119: 34-37

Nakayama A; Koyama H (1987) A General Similarity Transformation For Combined Free and Forced Convection Flows within a Fluid-Saturated Porous Medium. J Heat Transfer 109: 1041-1045

Nakayama A; Koyama H (1991) Buoyancy Induced Flow of NonNewtonian Fluids Over a Non-Isothermal Body of Arbitrary Shape in a Fluid-Saturated Porous Medium. Appl Scientific Res 48: 55-70 